\title{
BMJ Open Non-steroidal anti-inflammatory drugs and the risk of atrial fibrillation: a population-based follow-up study
}

\author{
Bouwe P Krijthe, ${ }^{1,2}$ Jan Heeringa, ${ }^{1}$ Albert Hofman, ${ }^{1}$ Oscar H Franco, ${ }^{1}$ \\ Bruno H Stricker ${ }^{1,2,3}$
}

To cite: Krijthe BP,

Heeringa J, Hofman A, et al. Non-steroidal

anti-inflammatory drugs and the risk of atrial fibrillation: a population-based follow-up study. BMJ Open 2014;4: e004059. doi:10.1136/ bmjopen-2013-004059

- Prepublication history for this paper is available online. To view these files please visit the journal online (http://dx.doi.org/10.1136/ bmjopen-2013-004059).

Received 17 September 2013 Revised 3 January 2014 Accepted 31 January 2014

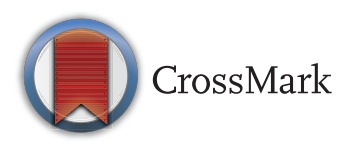

${ }^{1}$ Department of Epidemiology, Erasmus Medical Center, Rotterdam, The Netherlands

${ }^{2}$ Netherlands Consortium for Healthy Aging (NCHA), The Netherlands ${ }^{3}$ Inspectorate of Health Care, Hague, The Netherlands

Correspondence to Dr Bruno H Stricker; b.stricker@erasmusmc.nl

\section{ABSTRACT}

Objective: To investigate the association of nonsteroidal anti-inflammatory drugs (NSAIDs) and the risk of atrial fibrillation in a prospective communitybased follow-up study of elderly individuals with uniform case assessment and data on potential confounders.

Design: Data came from the population-based followup study, the Rotterdam Study.

Participants: The study comprised 8423 participants without atrial fibrillation at baseline.

Main outcome measures: Atrial fibrillation was ascertained from ECG assessments as well as medical records. Use of NSAIDs was obtained from automated prescription records by linkage with participating pharmacies. We used Cox proportional hazards models to study the association between NSAID drug use and atrial fibrillation. Use of NSAIDs was included in the model as a time-varying variable.

Results: At baseline, the mean age of the study population was 68.5 years (SD: 8.7 ) and $58 \%$ were women. During a mean follow-up of 12.9 years, 857 participants developed atrial fibrillation. Current use of NSAIDs was associated with increased risk compared with never-use (HR 1.76, 95\% $\mathrm{Cl} 1.07$ to 2.88). Also, recent use (within 30 days after discontinuation of NSAIDs) was associated with an increased risk of atrial fibrillation compared with never-use (HR 1.84, 95\% Cl 1.34 to 2.51 ) adjusted for age, sex and several potential confounders.

Conclusions: In this study, use of NSAIDs was associated with an increased risk of atrial fibrillation. Further studies are needed to investigate the underlying mechanisms behind this association.

\section{INTRODUCTION}

Atrial fibrillation (AF) is a common cardiac arrhythmia in the elderly, which is associated with increased morbidities (eg, stroke ${ }^{1}$ and heart failure ${ }^{2}$ ), increased mortality and reduced life expectancy. ${ }^{3-5}$ Several drugs have been associated with an increased risk of AF including steroidal anti-inflammatory drugs. ${ }^{6}{ }^{7}$ Recently, some studies suggested

\section{Strengths and limitations of this study}

- In this study, we included follow-up data from the Rotterdam Study, which is based in the general population and contains detailed information on drug exposure. Compared with previous database studies, we were able to use more detailed information for a range of potential confounders and to adjust for established risk factors of atrial fibrillation such as blood pressure and body mass index, and in a subsample for echocardiographic measures. Also, we were able to use a more detailed clinical assessment of atrial fibrillation. We used three different methods for case gathering and assessment, as we included every clinically recognised case from two different sources of medical records. In addition, we included repeated screening ECG assessments of the study population at the research centre.

- Compared with the previously published studies, however, our sample size was smaller. This might explain why some of our estimations did not reach statistical significance.

- Our study may have missed over-the-counter use of NSAIDs.

that non-steroidal anti-inflammatory drugs (NSAIDs) are also associated with a higher risk of $\mathrm{AF}^{8-10}$ NSAIDs are inhibitors of cyclooxygenase, and are widely used to treat inflammatory conditions and pain. Use of NSAIDs has previously been associated with a higher risk of myocardial infarction, stroke and heart failure. ${ }^{11-14}$ Moreover, NSAID use has been associated with increased cardiac dimensions such as left ventricular enddiastolic and systolic dimensions obtained with echocardiography. ${ }^{15}$

The previous studies investigating the association between NSAIDs and AF are mainly retrospective case-control studies or claims database studies with limited availability of potential confounders. Therefore, the objective of this study was to investigate whether 
use of NSAIDs is associated with $\mathrm{AF}$ in a prospective population-based cohort study with precise data on incident $\mathrm{AF}$, complete information on drug use and potential confounders.

\section{METHODS}

\section{Study population}

The current study was performed within the Rotterdam Study (RS), a population-based prospective cohort study, designed to examine the onset of, and risk factors for, disease in older adults. For this study, we combined participants from RS cohort 1 (RS-I), which started with a baseline visit between 1990 and 1993 ( $\mathrm{n}=7983)$, and from RS cohort 2 (RS-II), which started with a baseline visit between 2000 and $2001(\mathrm{n}=3011) \cdot{ }^{16}$ All participants aged 55 years and over who lived in the Ommoord district of Rotterdam, The Netherlands, were invited to participate. At baseline, participants were interviewed at home and examined at the research centre, which included a $10 \mathrm{~s}$, 12-lead resting ECG. From that visit onwards, participants were followed continuously and re-examined. For the current study, we used data from three follow-up examination rounds (1993-1995, 1997-1999 and 2002-2004) for RS-I, and from one re-examination round (2004-2005) for RS-II. Information on the presence and occurrence of disease at baseline and during follow-up is available by integrating information gathered by general practitioners in the study area. ${ }^{17}$ General practitioners in the Netherlands have a central and coordinating position in the Dutch Health Care system. They register all diagnoses available from their own work and from the work from physicians in the hospital and the outpatient clinic. Information on vital status was obtained on a regular basis from the Central Register of Population of the municipality of Rotterdam, from collaborating general practitioners, and by collecting information during follow-up examination rounds. For the participants for whom information remained missing, the Central Registry of Genealogy of the Netherlands was consulted. This national institute receives population registry records of all inhabitants of the Netherlands who have died. All participants were followed from their baseline ECG assessment without AF until the date of diagnosis of incident $\mathrm{AF}$, the date of death, loss to follow-up or end of the study period on 1 January 2009, whichever came first.

\section{AF assessment}

Prevalent and incident AF was ascertained using three methods. ${ }^{18}$ At baseline and at each follow-up examination, an ECG was recorded, stored digitally and analysed by the modular ECG analysis system (MEANS). ${ }^{19-21}$ Notably, MEANS is characterised by high sensitivity $(96.6 \%)$ and high specificity $(99.5 \%)$ in coding arrhythmias. ${ }^{19}$ To verify the diagnosis of AF, all ECGs with a diagnosis of $\mathrm{AF}$, atrial flutter or any other rhythm disorder were coded independently by two research physicians who were blinded to the MEANS diagnosis. The judgement of a cardiologist was taken as decisive in those cases in which disagreement persisted between the coding physicians. In addition, information was obtained from the treating general practitioners, which included their own results as well as results from medical specialists practising in hospitals and outpatient clinics. Patients were considered as a case of $\mathrm{AF}$ after diagnosis by a medical specialist or diagnosis by a general practitioner with ascertainment from an ECG. Finally, information was obtained from a Nationwide Medical Registry (LMR) of all hospital discharge diagnoses. The date of incident $\mathrm{AF}$ was defined as the date of the first occurrence of symptoms with subsequent ECG verification. We did not distinguish between $\mathrm{AF}$ and atrial flutter when we identified cases because both conditions are very similar with respect to risk factors and consequences. ${ }^{22} 23$

\section{Drug exposure}

Complete information on all filled prescriptions for all participants of the RS was obtained in an automated format from the collaborating pharmacies. This included the product name, international nonproprietary name, Anatomical Therapeutic Chemical code, total number of delivered units (eg, tablets or capsules), prescribed daily number of units, date of delivery and drug dosage. The duration of a prescription was calculated as the total number of delivered units divided by the prescribed daily number of units. The prescribed daily drug dosage was expressed in defined daily dose units, the recommended daily dosage of a drug taken by adults for the main indication of the drug by the WHO. $^{24}$

\section{Covariable assessment}

Body mass index (BMI) was calculated by dividing body weight in kilograms by squared height in metres. Blood pressure was measured twice at the right upper arm with a random zero mercury sphygmomanometer in the sitting position. The systolic and diastolic blood pressures were calculated as the average of the two consecutive measurements. Serum total cholesterol and high-density lipoprotein (HDL) cholesterol levels were measured with an automated enzymatic method. The presence of a myocardial infarction is based on clinical information from the medical records. For participants of the original RS-I cohort, the presence of myocardial infarction at baseline is based on verification of either self-reported myocardial infarction or ECG abnormalities indicative of prior myocardial infarction. In all subsequent cohorts and during follow-up, the medical records of all participants are screened, regardless of their self-reported history or ECG abnormalities. This information was independently validated by two cardiovascular research physicians. ${ }^{17}$ In cases in which the research physicians disagreed, judgement of a medical specialist was considered decisive. ${ }^{17}$ Heart failure was assessed using a validated score based on the definition of heart failure by the European Society of 
Cardiology. ${ }^{25}{ }^{26}$ Prevalent diabetes mellitus was defined as a preload or postload serum glucose level of $>11 \mathrm{mmol} / \mathrm{L}$ determined at a research visit or the use of hypoglycaemic medication. ${ }^{27}$

\section{Statistical analysis}

To study the association between NSAID drug use and $\mathrm{AF}$, we used Cox proportional hazards models. Use of NSAIDs was included in the model as a time-varying covariable as described earlier. ${ }^{28}$ During follow-up, each time an event of AF occurred (index date), we determined the exposure to NSAIDs in each case and in the remainder of the cohort of participants on the same day of follow-up. Persons were considered as current users of an NSAID if an event date fell between the start date and end date of a prescription. If a person had previously used an NSAID but no longer used the drug on an event date, they were considered as a past user. Participants were categorised as current users (distinguished into: use $\leq 14 ; 15-30$; $>30$ days), past users (distinguished into: stopped $\leq 30 ; 31-180 ;>180$ days ago) and never users. Persons who had not used an NSAID at any time during the study period were categorised as 'never users'. Also, we studied whether the prescribed dosage of current users was associated with the risk of AF. All analyses were adjusted for age at index date and sex. In a second model, we additionally adjusted for established risk factors for AF including baseline values of systolic blood pressure, diastolic blood pressure, BMI, total and HDL cholesterol, smoking status and timedependent covariables: blood pressure lowering drug use, history of myocardial infarction, presence of heart failure and presence of diabetes. Missing data for these covariates were imputed using an expectation maximisation algorithm. As NSAID use has been associated with an increased left ventricular end-diastolic dimension obtained by echocardiography, ${ }^{15}$ we hypothesised that this could explain the association of NSAID use with AF. Therefore, we additionally adjusted for left ventricular end-diastolic dimension in a sensitivity analysis. In this analysis, participants were followed from the date of the echocardiographic examination (fourth research centre visit RS-I: 2002-2004, second research centre visit RS-II: 2004-2005) onwards $(\mathrm{n}=4919)$.

\section{RESULTS}

The total population of cohorts RS-I and RS-II consisted of 10994 participants (7983 from the first cohort and 3011 from the second cohort). Of these, 459 participants had been diagnosed with AF at the baseline of the study and 2112 had missing information on AF status or drug exposure and were therefore excluded from the analysis. This resulted in a population for analysis of 8423 participants. At baseline, the mean age of the study population was 68.5 years (SD 8.7), of whom the majority were women $(58.6 \%$; table 1$)$. During a mean follow-up of 12.9 years, 857 participants developed AF.
Table 1 Baseline characteristics of study population $(\mathrm{N}=8423)$

\begin{tabular}{lc}
\hline & Mean (SD)/N (\%) \\
\hline Age (years) & $68.5(8.7)$ \\
Female sex & $4940(58.6)$ \\
Systolic blood pressure $(\mathrm{mm} \mathrm{Hg})$ & $140(22)$ \\
Diastolic blood pressure $(\mathrm{mm} \mathrm{Hg})$ & $75(11)$ \\
Blood pressure lowering drugs & $2828(33.6)$ \\
Body mass index $\left(\mathrm{kg} / \mathrm{m}^{2}\right)$ & $26.5(3.8)$ \\
Total cholesterol & $6.4(1.2)$ \\
HDL cholesterol & $1.4(0.4)$ \\
Current smoker & $1845(21.9)$ \\
Past smoker & $3643(43.3)$ \\
History of myocardial infarction & $791(9.4)$ \\
Presence of congestive heart failure & $160(1.9)$ \\
Presence of diabetes mellitus & $864(10.4)$ \\
\hline HDL, high-density lipoprotein. &
\end{tabular}

At the moment of diagnosis of AF, 261 cases had never used NSAIDs, 554 had used NSAIDs in the past, and 42 were currently using NSAIDs. Of these current users, the majority $(n=29)$ used a non-selective NSAID, five participants used a COX-1 selective NSAID, and seven used a COX-2 selective NSAID.

\section{NSAIDs and the risk of AF}

Current use of NSAIDs for 15-30 days was associated with an increased risk of AF compared with never use (HR 1.76, 95\% CI 1.07 to 2.88 adjusted for age, sex and cardiovascular risk factors; table 2). Also, recent past use of NSAIDs, being use within the preceding 30 days, was associated with an increased risk of AF (HR 1.84, 95\% CI 1.34 to 2.51 adjusted for age, sex and cardiovascular risk factors). Although higher dosages seemed to be associated with a higher risk of $\mathrm{AF}$, this did not reach statistical significance.

In a sensitivity analysis, we additionally adjusted for left ventricular end-diastolic dimension (table 3). In this analysis, left ventricular end-diastolic dimension was associated with an increased risk of AF (HR per SD increment: $1.17,95 \%$ CI 1.01 to 1.35 adjusted for age, sex, NSAID use and cardiovascular risk factors). However, even after adjusting for left ventricular dimension, current NSAID use was associated with AF (HR 1530 days: $3.21,95 \%$ CI 1.21 to 8.48 , adjusted for age, sex and left ventricular end-diastolic dimension and cardiovascular risk factors) compared with never use. Similar results were found adjusting for left ventricular end systolic dimension or left atrial size (table 3 ).

\section{DISCUSSION}

Our results suggest that NSAID use is associated with a higher risk of AF. Current use and recent past use were associated with a higher risk of AF, adjusted for age, sex and cardiovascular risk factors. Also, after additional 
Table 2 The association of NSAID use with risk of atrial fibrillation

\begin{tabular}{|c|c|c|c|c|c|}
\hline & \multirow[b]{2}{*}{$\mathbf{n}$} & \multicolumn{2}{|c|}{ Model 1* } & \multicolumn{2}{|c|}{ Model $2 \dagger$} \\
\hline & & $\overline{\mathrm{HR}}$ & $95 \% \mathrm{Cl}$ & $\overline{\mathrm{HR}}$ & $95 \% \mathrm{Cl}$ \\
\hline Never use & 261 & 1 & (Ref.) & 1 & (Ref.) \\
\hline \multicolumn{6}{|l|}{ Current use (days) } \\
\hline$\leq 14$ & 17 & 1.08 & (0.66 to 1.76$)$ & 0.96 & (0.59 to 1.58$)$ \\
\hline $15-30$ & 17 & 1.99 & (1.22 to 3.26$)$ & 1.76 & (1.07 to 2.88$)$ \\
\hline$>30$ & 8 & 1.00 & (0.50 to 2.03 ) & 0.84 & (0.41 to 1.70$)$ \\
\hline \multicolumn{6}{|l|}{ Past use (days) } \\
\hline$\leq 30$ & 47 & 2.01 & (1.47 to 2.75 ) & 1.84 & (1.34 to 2.51 ) \\
\hline $31-180$ & 76 & 1.10 & (0.85 to 1.42$)$ & 1.00 & (0.77 to 1.29$)$ \\
\hline$>180$ & 431 & 1.11 & (0.94 to 1.30$)$ & 1.04 & (0.88 to 1.22$)$ \\
\hline No use & 815 & 1 & (Ref.) & 1 & (Ref.) \\
\hline \multicolumn{6}{|l|}{ Dosage current use } \\
\hline Low-medium dosage (PDD $\leq 1$ ) & 24 & 1.09 & (0.73 to 1.64$)$ & 0.97 & (0.65 to 1.46$)$ \\
\hline High dosage $(P D D>1.0)$ & 18 & 1.33 & (0.83 to 2.11$)$ & 1.27 & (0.80 to 2.03 ) \\
\hline
\end{tabular}

${ }^{*}$ Adjusted for age at index date and sex.

†Additionally adjusted for baseline values of: systolic blood pressure, diastolic blood pressure, body mass index, total and HDL cholesterol, smoking status. Time dependent covariates include: blood pressure lowering drugs, history of myocardial infarction, presence of heart failure, prevalent diabetes.

HDL, high-density lipoprotein; $n$, number of atrial fibrillation cases; NSAID, non-steroidal anti-inflammatory drug; PDD, prescribed daily dosage.

adjustment for echocardiographic parameters, current NSAID use was associated with an increased risk of AF.

Previously, a case-control study from the UK with data from the General Practice Research Database showed that current use of NSAIDs was associated with an increased risk of $\mathrm{AF}^{8}$ These results were later replicated by Schmidt et $a t$ in a population-based case-control study in Denmark. They showed that use of NSAIDs was associated with an increased risk of AF. Furthermore, their results suggested that the risk of AF was strongest for COX-2 inhibitors, which was later confirmed by others. ${ }^{10}$ Our results support most of these previously published results but also suggest that the increased risk occurs shortly after starting treatment and may disappear over time. Schmidt et al also showed that longer current use was not associated with an increased risk of
AF. There are two possible explanations for these results. First, it is possible that these results can be explained by 'depletion of susceptibles' if those with symptoms discontinue drug use. Also, it may be that the acute effects associated mainly with NSAID use, which leads to the development of AF. The fact that recent past users who, according to their prescription, also stopped using NSAIDs in the preceding 30 days had a higher risk of AF can be explained if symptoms lead to discontinuation of the NSAID. Also, it is possible that they might still be using it at the index date or still have active drug levels.

Several mechanisms might explain the association of NSAIDs with risk of AF. It is possible that NSAIDs play a causal role in the development of $\mathrm{AF}$, as they inhibit cyclo-oxygenase. ${ }^{29}$ Cyclo-oxygenase enzymes are expressed in kidney tissue. ${ }^{29}$ Inhibition of these enzymes may lead to

Table 3 The association of NSAID use with risk of atrial fibrillation, additionally adjusted for left ventricular end-diastolic dimension at baseline $(\mathrm{N}=4919)$

\begin{tabular}{|c|c|c|c|c|c|}
\hline & \multirow[b]{2}{*}{$\mathbf{n}$} & \multicolumn{2}{|c|}{ Model $1^{*}$} & \multicolumn{2}{|c|}{ Model 2† } \\
\hline & & HR & $95 \% \mathrm{Cl}$ & HR & $95 \% \mathrm{Cl}$ \\
\hline Never use & 24 & 1 & (Ref.) & 1 & (Ref.) \\
\hline \multicolumn{6}{|c|}{ Current use (days) } \\
\hline$\leq 14$ & 0 & NA & (NA) & NA & (NA) \\
\hline $15-30$ & 5 & 3.61 & (1.37 to 9.50$)$ & 3.21 & (1.22 to 8.48$)$ \\
\hline$>30$ & 3 & 1.97 & (0.59 to 6.56$)$ & 1.71 & (0.51 to 5.70$)$ \\
\hline \multicolumn{6}{|c|}{ Past use (days) } \\
\hline$\leq 30$ & 6 & 1.61 & (0.66 to 3.94$)$ & 1.47 & (0.60 to 3.62 ) \\
\hline $31-180$ & 13 & 1.14 & (0.58 to 2.24$)$ & 1.03 & (0.52 to 2.03 ) \\
\hline$>180$ & 128 & 1.42 & (0.92 to 2.20 ) & 1.32 & (0.85 to 2.06 ) \\
\hline
\end{tabular}

${ }^{*}$ Adjusted for age at index date, sex and left ventricular end-diastolic dimension.

†Additionally adjusted for baseline values of: systolic blood pressure, diastolic blood pressure, body mass index, total and HDL cholesterol, smoking status and left ventricular end-diastolic dimension. Time dependent covariates include: blood pressure lowering drugs, history of myocardial infarction, presence of heart failure, prevalent diabetes.

HDL, high-density lipoprotein; $n$, number of atrial fibrillation cases; NA, not applicable; NSAID, non-steroidal anti-inflammatory drug. 
an increase in blood pressure due to fluid retention, increased peripheral resistance and attenuation of diuretic and antihypertensive drug effects. ${ }^{9}{ }^{29}$ It was shown that current NSAID use is associated with increased enddiastolic and end-systolic dimension obtained with echocardiography in the first 14 days of treatment with NSAIDs, and with end-diastolic dimension alone after longer use. ${ }^{15}$ Possibly these changes in left ventricular dimensions could explain part of the association between NSAIDs and atrial fibrillation. In a sensitivity analysis in patients for whom echocardiography was available, we adjusted for baseline left ventricular end-diastolic dimension in a subsample of our population. In this analysis, left ventricular end-diastolic dimension was indeed associated with a higher risk of AF. After adjustment for left ventricular end-diastolic dimension, NSAID use remained associated with the risk of AF. However, as end-diastolic diameter at the moment of AF was not available, it is possible that NSAID use through fluid retention and increasing end-diastolic of end-systolic dimension increases the risk of AF. Moreover, COX inhibition may lead to fluctuation of serum potassium by decreased excretion in the distal nephron. ${ }^{29}$ Possibly these adverse renal effects may trigger AF. ${ }^{6}$ However, it is also possible that NSAID use is an indicator of the presence of the underlying inflammatory disease. These underlying inflammatory conditions might be associated with the risk of $\mathrm{AF}^{30}$

Our study has several strengths. We included follow-up data from the RS, which is based on the general population and contains detailed information on drug exposure. Compared with previous database studies, we were able to use more detailed information for a range of potential confounders and to adjust for established risk factors of AF such as blood pressure and BMI, and in a subsample for echocardiographic measures. Also, we were able to use a more accurate clinical assessment of AF. We used three different methods for case gathering and assessment, as we included every clinically recognised case from two different sources of medical records. In addition, we included repeated screening ECG assessments of the study population at the research centre. Compared with the previously published studies, however, our sample size was smaller. This might explain why some of our estimations did not reach statistical significance. Also, we only had data available prescribed on NSAIDs and did not have information on the indication for the prescription nor on the use of NSAIDs that were bought without a prescription. Finally, we were not able to categorise according to $\mathrm{COX}$ selectivity because of the low number of users of COX-1 and COX-2 inhibitors.

In conclusion, we found that use of NSAIDs is associated with an increased risk of AF. Current use and recent past use were especially associated with a higher risk of AF, adjusted for age, sex and cardiovascular risk factors. The underlying mechanism behind this association deserves further attention.
Acknowledgements The authors thank all the participants and staff of the Rotterdam Study, as well as the general practitioners and pharmacists of the Ommoord district for help with data collection and validation.

Contributors The authors BPK, JH and BHS were responsible for the study concept and design. BPK, JH, OHF and BHS were involved in the analysis and interpretation of the data. All the authors were responsible for the drafting and revision of the manuscript and also read and approved it.

Funding The Rotterdam Study is supported by the Erasmus Medical Center and Erasmus University Rotterdam; The Netherlands Organisation for Scientific Research (NWO); The Netherlands Organisation for Health Research and Development (ZonMw); the Research Institute for Diseases in the Elderly (RIDE); The Netherlands Heart Foundation; the Ministry of Education, Culture and Science; the Ministry of Health Welfare and Sports; the European Commission (DG XII); and the Municipality of Rotterdam. Oscar H. Franco works in ErasmusAGE, a Center for aging research across the life course funded by Nestlé Nutrition (Nestec Ltd.); Metagenics Inc and AXA. Nestlé Nutrition (Nestec Ltd); Metagenics Inc and AXA had no role in the design and conduct of the study; collection, management, analysis and interpretation of the data and preparation, review or approval of the manuscript.

\section{Competing interests None.}

Ethics approval The medical ethics committee of the Erasmus Medical Center, Rotterdam, approved the study, and all participants gave informed consent to participate in the study and to obtain information from treating physicians separately.

Provenance and peer review Not commissioned; externally peer reviewed.

Data sharing statement No additional data are available.

Open Access This is an Open Access article distributed in accordance with the Creative Commons Attribution Non Commercial (CC BY-NC 3.0) license, which permits others to distribute, remix, adapt, build upon this work noncommercially, and license their derivative works on different terms, provided the original work is properly cited and the use is non-commercial. See: http:// creativecommons.org/licenses/by-nc/3.0/

\section{REFERENCES}

1. Marini C, De Santis F, Sacco S, et al. Contribution of atrial fibrillation to incidence and outcome of ischemic stroke: results from a population-based study. Stroke 2005;36:1115-19.

2. Wang TJ, Larson MG, Levy D, et al. Temporal relations of atrial fibrillation and congestive heart failure and their joint influence on mortality: the Framingham Heart Study. Circulation 2003;107:2920-5

3. Stewart S, Hart CL, Hole DJ, et al. A population-based study of the long-term risks associated with atrial fibrillation: 20-year follow-up of the Renfrew/Paisley study. Am J Med 2002;113:359-64.

4. Miyasaka Y, Barnes ME, Bailey KR, et al. Mortality trends in patients diagnosed with first atrial fibrillation: a 21-year community-based study. J Am Coll Cardiol 2007;49:986-92.

5. Benjamin EJ, Wolf PA, D'Agostino RB, et al. Impact of atrial fibrillation on the risk of death: the Framingham Heart Study. Circulation 1998;98:946-52.

6. van der Hooft CS, Heeringa J, van Herpen G, et al. Drug-induced atrial fibrillation. J Am Coll Cardiol 2004;44:2117-24.

7. van der Hooft CS, Heeringa J, Brusselle GG, et al. Corticosteroids and the risk of atrial fibrillation. Arch Intern Med 2006;166:1016-20.

8. De Caterina R, Ruigomez A, Rodriguez LA. Long-term use of anti-inflammatory drugs and risk of atrial fibrillation. Arch Intern Med 2010;170:1450-5

9. Schmidt M, Christiansen CF, Mehnert F, et al. Non-steroidal anti-inflammatory drug use and risk of atrial fibrillation or flutter: population based case-control study. BMJ 2011;343:d3450.

10. Back M, Yin L, Ingelsson E. Cyclooxygenase-2 inhibitors and cardiovascular risk in a nation-wide cohort study after the withdrawal of rofecoxib. Eur Heart J 2012;33:1928-33.

11. Haag MD, Bos MJ, Hofman A, et al. Cyclooxygenase selectivity of nonsteroidal anti-inflammatory drugs and risk of stroke. Arch Intern Med 2008;168:1219-24.

12. Bresalier RS, Sandler RS, Quan $\mathrm{H}$, et al. Cardiovascular events associated with rofecoxib in a colorectal adenoma chemoprevention trial. N Engl J Med 2005;352:1092-102. 
13. Solomon SD, McMurray JJ, Pfeffer MA, et al. Cardiovascular risk associated with celecoxib in a clinical trial for colorectal adenoma prevention. N Engl J Med 2005;352:1071-80.

14. Nussmeier NA, Whelton AA, Brown MT, et al. Complications of the COX-2 inhibitors parecoxib and valdecoxib after cardiac surgery. N Engl J Med 2005;352:1081-91.

15. van den Hondel KE, Eijgelsheim M, Ruiter R, et al. Effect of short-term NSAID use on echocardiographic parameters in elderly people: a population-based cohort study. Heart 2011;97:540-3.

16. Hofman A, van Duijn CM, Franco OH, et al. The Rotterdam Study: 2012 objectives and design update. Eur J Epidemiol 2011;26:657-86.

17. Leening MJ, Kavousi M, Heeringa J, et al. Methods of data collection and definitions of cardiac outcomes in the Rotterdam Study. Eur J Epidemiol 2012;27:173-85.

18. Heeringa J, van der Kuip DA, Hofman A, et al. Prevalence, incidence and lifetime risk of atrial fibrillation: the Rotterdam study. Eur Heart J 2006;27:949-53.

19. Kors JA, van Herpen G, Wu J, et al. Validation of a new computer program for Minnesota coding. J Electrocardiol 1996;29(Suppl): 83-8.

20. van Bemmel JH, Kors JA, van Herpen G. Methodology of the modular ECG analysis system MEANS. Methods Inf Med 1990:29:346-53.

21. Willems JL, Abreu-Lima C, Arnaud P, et al. The diagnostic performance of computer programs for the interpretation of electrocardiograms. N Engl J Med 1991;325:1767-73.
22. Halligan SC, Gersh BJ, Brown RD Jr, et al. The natural history of lone atrial flutter. Ann Intern Med 2004;140:265-8.

23. Lelorier $\mathrm{P}$, Humphries $\mathrm{KH}$, Krahn A, et al. Prognostic differences between atrial fibrillation and atrial flutter. Am J Cardiol 2004;93:647-9.

24. WHO. Collaborating Centre for Drug Statistics Methodology, Guidelines for ATC classification and DDD assignment 2012. Oslo, 2011.

25. Remme WJ, Swedberg K; Task Force for the Diagnosis and Treatment of Chronic Heart Failure, European Society of Cardiology. Guidelines for the diagnosis and treatment of chronic heart failure. Eur Heart J 2001;22:1527-60.

26. Mosterd A, Hoes AW, de Bruyne MC, et al. Prevalence of heart failure and left ventricular dysfunction in the general population; the Rotterdam Study. Eur Heart J 1999;20:447-55.

27. Dehghan A, Kardys I, de Maat MP, et al. Genetic variation, C-reactive protein levels, and incidence of diabetes. Diabetes 2007:56:872-8.

28. Stricker BH, Stijnen T. Analysis of individual drug use as a timevarying determinant of exposure in prospective population-based cohort studies. Eur J Epidemiol 2010;25:245-51.

29. Whelton A. Renal aspects of treatment with conventional nonsteroidal anti-inflammatory drugs versus cyclooxygenase-2specific inhibitors. Am J Med 2001;110(Suppl 3A):33S-42S.

30. Lindhardsen J, Ahlehoff O, Gislason GH, et al. Risk of atrial fibrillation and stroke in rheumatoid arthritis: Danish nationwide cohort study. BMJ 344:e1257. 